\title{
Computation of dendritic microstructures using a level set method
}

\author{
Yung-Tae Kim ${ }^{1}$, Nigel Goldenfeld ${ }^{1}$, and Jonathan Dantzig ${ }^{2}$ \\ ${ }^{1}$ University of Illinois at Urbana-Champaign, Department of Physics \\ 1110 West Green Street, Urbana, IL, 61801 \\ ${ }^{2}$ University of Illinois at Urbana-Champaign, Department of Mechanical and Industrial Engineering \\ 1206 West Green Street, Urbana, IL, 61801
}

(October 22, 2018)

\begin{abstract}
We compute time-dependent solutions of the sharp-interface model of dendritic solidification in two dimensions by using a level set method. The steady-state results are in agreement with solvability theory. Solutions obtained from the level set algorithm are compared with dendritic growth simulations performed using a phase-field model and the two methods are found to give equivalent results. Furthermore, we perform simulations with unequal diffusivities in the solid and liquid phases and find reasonable agreement with the available theory.
\end{abstract}

PACS Numbers: 81.10.Aj, 68.70.+w, 81.30.Fb, 64.70.Dv

Various numerical approaches [1] have been developed to solve the difficult moving boundary problem that governs the growth of dendrites [5 7]. Unfortunately, the direct solution of the time-dependent Stefan problem is troublesome and usually requires front tracking and lattice deformation in order to contain the moving solidliquid interface, which is often very complicated topologically. In general, the methods developed to tackle the free-boundary problem have difficulty in handling topology changes, such as the merging and breaking of surfaces, and are usually not easily extendible to higher dimensions.

In order to avoid the difficulties associated with tracking a sharp interface, the phase-field model of solidification has been developed and is currently the most popular technique for simulating dendritic growth. The phasefield model avoids the computational difficulties associated with front tracking by introducing an auxiliary order parameter, or phase-field, $\psi(\mathbf{r}, t)$ that couples to the evolution of the thermal field. The dynamics of $\psi(\mathbf{r}, t)$ are designed to follow the evolving solidification front [8 11], which is defined by the zero level set $\psi(\mathbf{r}, t)=0$. Because the interface is never explicitly tracked, complicated topology changes are handled easily. Furthermore, the extension of the phase-field model to higher dimensions is straightforward.

Although phase-field models have been very useful in studying solidification patterns, there are still some limitations in this approach. The proper use of these models requires that an asymptotic analysis be performed in order to obtain a mapping between the parameters of the phase-field equations and the sharp-interface equations [12 14]. The asymptotics involve expanding the phase-field equations in some small parameter proportional to the interface width, $W$, and as a result, the phase-field model only reproduces the dynamics of the sharp-interface equations in the limit where the expansion parameter is sufficiently small. Computationally, the grid spacing must be small enough to resolve the interfa- cial region, which is on the order of $W$. This restriction is generally not a problem for the symmetric model of solidification (where the diffusivities in the solid and liquid phases are assumed to be the same) because it is possible to have $W$ on the order of the capillary length 114. However, phase-field asymptotics for unequal diffusivities can be problematic [15]; correction terms that are inconsistent with the sharp-interface equations are generated and non-monotonic behavior is required in the interfacial region, which requires extra grid resolution and hence slower computational performance. The generalization of the phase-field approach to handle discontinuous material properties requires a better understanding of the mapping between the phase-field model and the sharp-interface formulation in order to avoid problems with properly resolving the interface.

The level set method is a computational approach that has the capability of avoiding the above mentioned limitations of front tracking methods and phase-field models. This method, first introduced by Osher and Sethian [16], is conceptually similar to a phase-field model in that the solid-liquid interface is represented as the zero contour of a level set function, $\phi(\mathbf{r}, t)$, which has its own equation of motion. The movement of the interface is taken care of implicitly through an advection equation for $\phi(\mathbf{r}, t)$. Thus, topology changes and the extension of the method to higher dimensions can be handled in a straightforward manner. Unlike the phase-field model, there is no arbitrary interface width introduced in the level set method; the sharp-interface equations can be solved directly and, as a result, no asymptotics are required. Discontinuous material properties can also be dealt with in a simple manner.

The level set method has been applied to several problems involving moving boundaries 17 19], including solidification. Prior work on dendritic growth includes an application of the method to a boundary integral formulation 20 as well as the direct solution of the sharpinterface equations [21]. While these simulations have 
reproduced the qualitative features of dendrites, as well as some quantitatively accurate solutions to exactly soluble problems, some of the simulations of anisotropic dendritic growth were not necessarily converged [21]. Furthermore, the results were not compared with theoretical predictions of dendritic growth.

In this paper, we demonstrate that the level set method can be used to solve the free-boundary problem for solidification to calculate quantitatively accurate solutions for dendritic growth. We present results from simulations in two dimensions and show that the solutions converge to the steady-state predicted by microscopic solvability theory. Time-dependent results are also compared with calculations using a phase-field model and good agreement is found for all times. Furthermore, we perform simulations with unequal diffusivities (a case which is not yet possible with phase-field models) and find that the prediction of Barbieri and Langer [22] provides a fair quantitative fit to our results.

The solidification of a pure substance is described by a free-boundary problem for the temperature in the solid and liquid phases, and the position of the interface between them:

$$
\begin{aligned}
\partial_{t} u & =D \nabla^{2} u \\
V_{n} & =\left(D \partial_{n} u\right)_{\text {Solid }}-\left(D \partial_{n} u\right)_{L i q u i d} \\
u_{i} & =-d(\theta) \kappa-\beta(\theta) V_{n}
\end{aligned}
$$

The temperature $T$ has been rescaled as a dimensionless thermal field $u=\left(T-T_{m}\right) /\left(L / C_{p}\right)$, where $T_{m}, L$, and $C_{p}$ represent the melting temperature, the latent heat of fusion, and the specific heat at constant pressure, respectively. The thermal diffusivity, $D$, can be different in the solid and liquid phases. Eq. 2 describes energy conservation at the solid-liquid interface, where $V_{n}$ is the local outward normal interface velocity and $\partial_{n}$ refers to the outward normal derivative at the interface. Finally, Eq. 3 is known as the Gibbs-Thomson condition and describes the deviation of the interface temperature, $u_{i}$, from equilibrium due to the local curvature, $\kappa$, and interface kinetics. $d(\theta)=\gamma(\theta) T_{m} C_{p} / L^{2}$ is the anisotropic capillary length, proportional to the surface tension $\gamma(\theta)$, and $\beta(\theta)$ is the anisotropic kinetic coefficient. Here we assume that $\beta(\theta)=0$ and that the capillary length has the form $d(\theta)=d_{o}(1-15 \epsilon \cos 4 \theta)$, where $\epsilon$ is the anisotropy strength and $\theta$ is the angle between the local normal vector at the interface, $\vec{n}$, and the $x$-axis.

We solve the above free-boundary problem by using a level set algorithm, which involves the following steps: i) advancing the interface, ii) reinitializing the level set function to be a signed distance function, and iii) solving for the new thermal field. The general level set method is described below. We wish to note that in our simulations we implement a localized level set method, described in detail in Ref. 23], in which calculations of $\phi$ are performed only in a narrow region around the interface. We have not yet made an attempt to make our algorithm more computationally efficient by using adaptive mesh refinement.

i) Advancing the interface. The level set function is defined as the signed normal distance from the solid-liquid interface such that $\phi$ is positive in the liquid phase, negative in the solid phase, and zero at the interface. $\phi$ satisfies the pure advection equation

$$
\frac{\partial \phi}{\partial t}+F|\nabla \phi|=0
$$

Integrating Eq. 4 for one timestep results in moving the contours of $\phi$ along the directions normal to the interface according to the velocity field $F$, which varies in space. $F$ is constructed to be an extension of the interface velocity, $V_{n}$, such that $F=V_{n}$ for points on the interface and the lines of constant $F$ are normal to the interface. Thus, advecting $\phi$ according to Eq. 1 moves the front with the correct velocity.

Rather than using a partial differential equation to generate $F$ (as in Refs. 21, 23), we construct $F$ in the following manner: $\phi$ represents the normal distance from the solidification front, so the value of $\phi$ at each gridpoint on the computational lattice can be used to locate a particular point on the interface. If $\vec{x}_{g}$ is the location of the gridpoint, the associated point on the interface is at $\vec{x}_{i}=\vec{x}_{g}-\phi \vec{n}$, where the normal vector $\vec{n}=\nabla \phi /|\nabla \phi|$. The temperature at $\vec{x}_{i}$ is then calculated by using Eq. 3; $\theta$ is easily found from $\vec{n}$, and the curvature, $\kappa=\nabla \cdot \vec{n}$, is interpolated at $\vec{x}_{i}$ from values of $\kappa$ at neighboring gridpoints. $\vec{n}$ and $\kappa$ are calculated using standard, centered finite difference approximations to the partial derivatives of $\phi$. Next, values of $u$ are interpolated in both the liquid and solid phases, a distance $\Delta x$ (the size of the grid spacing) away from $\vec{x}_{i}$ along the normal direction. These two interpolated temperatures are used along with $u_{i}$ to approximate the difference in the normal derivative of $u$ at $\vec{x}_{i}$ and thus find $V_{n}$ (Eq. 3). Because $\vec{x}_{i}$ and $\vec{x}_{g}$ lie in the same line normal to the interface, the value of $F$ at $\vec{x}_{g}$ is simply $V_{n}$. The field $F$ can be determined at all gridpoints in this way.

After $F$ is known, the interface can be advanced one timestep. For stability, we discretize Eq. 4 using a 5th order WENO (weighted essentially non-oscillatory) scheme in space and a 3rd order Runge-Kutta scheme in time [24]. However, the overall accuracy of our algorithm is second order in space and first order in time.

ii) Reinitialization. After solving Eq. 4 for one timestep, the level set function will no longer be equal to the distance away from the interface. It is necessary to reinitialize $\phi$ to be a signed distance function. This step is accomplished by solving

$$
\frac{\partial \phi}{\partial t}+S(\phi)[|\nabla \phi|-1]=0
$$

to steady state. $S(\phi)$ takes on the value +1 in the liquid phase, -1 in the solid phase, and is zero at the interface. We typically iterate Eq. 5 three times in order to obtain 
an accurate distance function. Like Eq. 14, this equation is discretized using a 5th order WENO scheme in space and a 3rd order Runge-Kutta scheme in time [24].

iii) Solving for the new thermal field. The thermal field is updated by solving Eq. 1 using a modified CrankNicolson scheme. Different diffusivities in the two phases can be taken into account by simply noting the sign of the level set function and using the appropriate diffusion coefficient in the finite difference stencil. Special care has to be taken for gridpoints near the interface. If $|\phi| \leq \Delta x$, the level set function is used to determine whether the front intersects the stencil and, if so, interpolate where the interface crosses the stencil. The stencil is then modified to take into account the location of the interface and the Gibbs-Thomson condition.

We compute four-fold symmetric dendrites in a $L \times L$ square box using the procedure described above. Solidification is initiated by a small quarter disk of radius $R_{o}$ in the lower left-hand corner of the box. The initial level set function is $\phi(x, y)=\sqrt{x^{2}+y^{2}}-R_{o}$, where $x$ and $y$ are the usual Cartesian coordinates. The initial temperature is $u=0$ in the solid and decays exponentially away from the interface to $u=-\Delta$ as $\vec{x} \rightarrow \infty$, where the far-field undercooling is $\Delta=\left(T_{m}-T_{\infty}\right) /\left(L / C_{p}\right)$ and $T_{\infty}$ is the temperature far ahead of the solidification front in the liquid.

Eqs. 1-3 have been studied extensively to determine the steady state features of dendritic growth [6.67. According to microscopic solvability theory, these equations admit a family of discrete solutions. Only the fastest growing of this set of solutions is stable. This solution is the dynamically selected "operating state" for the dendrite and corresponds to a unique tip shape and velocity. Recent calculations of dendritic growth using phase-field models have been found to be in good agreement with the predictions of microscopic solvability theory [14,25]. We observe similar agreement with the use of the level set algorithm and obtain results that are within a few percent of theoretical predictions. Figure 1 shows the tip velocity of the dendrite versus time for computations at undercoolings of $\Delta=0.65$ and 0.55 . For all of these simulations $D=1, d_{o}=0.5, \beta=0, R_{o}=15$, and $\epsilon=0.05$. For the $\Delta=0.65$ simulation, $L=200, \Delta x=0.2$, and the timestep is chosen to be $\Delta t=0.002$. For the $\Delta=0.55$ simulation, $L=800, \Delta x=0.4$, and $\Delta t=0.008$. To ensure grid convergence, $\Delta x$ and $\Delta t$ were refined until the steady-state tip velocity did not vary by more than $2 \%$.

We also compare our level set results with simulations of dendritic growth performed using a phase-field model. Although calculations using phase-field models have been compared with a steady-state theory, there have been no comparisons made between time-dependent phase-field calculations and time-dependent solutions of the sharp-interface equations for multi-dimensional dendritic growth. The phase-field model calculations presented here were performed using a special adaptive mesh algorithm, as described in Ref. 25. The tip velocity data from the phase-field model and level set method

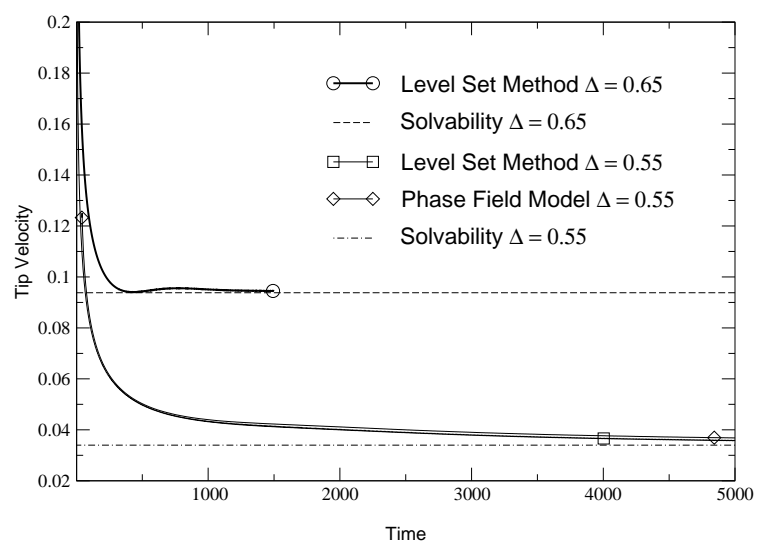

FIG. 1. Time evolution of the tip velocity for simulations at $\Delta=0.65$ and 0.55 .

at $\Delta=0.55$ are in excellent agreement with each other (within 3\%), as shown in Figure 1. Similar agreement is found in the dendritic shapes for these simulations, presented at time $=9400$ in Figure 2. These comparative results, combined with the recent demonstration of the equivalence of various phase-field models [26], provide an excellent foundation for the validity of the phase-field approach in simulating solidification microstructures.

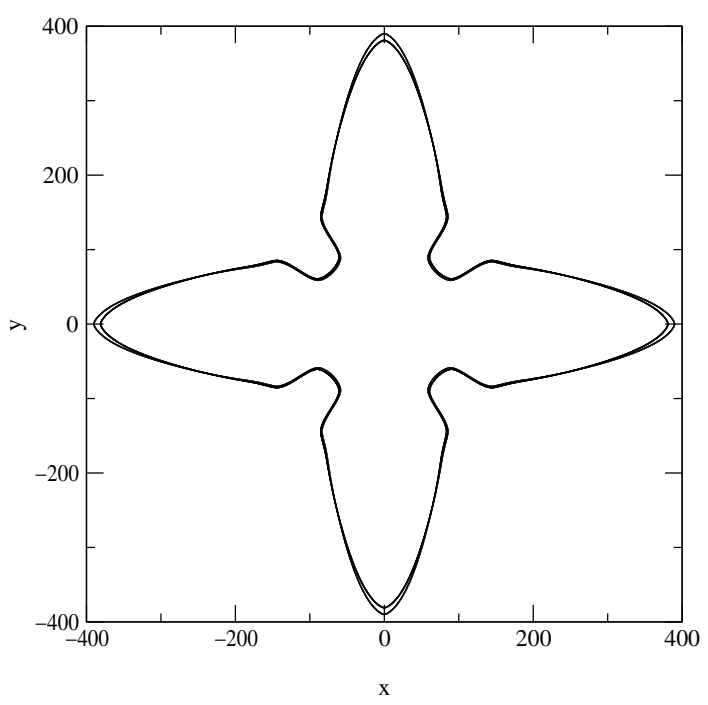

FIG. 2. Comparison of dendritic shapes computed from the level set method and phase-field model for $\Delta=0.55$, shown at time $=9400$.

The results presented here so far have used $D_{S}=D_{L}$, where $D_{S}$ and $D_{L}$ are the diffusivities in the solid and liquid phases, respectively. With our level set algorithm, we can also investigate the more general case where the 
diffusivities are unequal. We performed additional simulations at $\Delta=0.65$ with $D_{S}=0.75,0.5,0.25$ and 0 while keeping $D_{L}=1$. The only available benchmark for the case of non-symmetric diffusion is the linearized solvability theory of Barbieri and Langer [22], which predicts

$$
\rho^{2} V \approx \frac{1+D_{S} / D_{L}}{2}\left(\rho^{2} V\right)_{D_{S} / D_{L}=1}
$$

where $\rho$ and $V$ are the steady-state tip radius and velocity, respectively. The values of $\rho^{2} V$ obtained from the level set simulations are compared with Eq. 6 in Figure 3. The fit is surprisingly good (an error of about $13 \%$ at $\left.D_{S} / D_{L}=0\right)$, considering Eq. 6 was obtained from a linearized theory in the limit of small undercoolings.

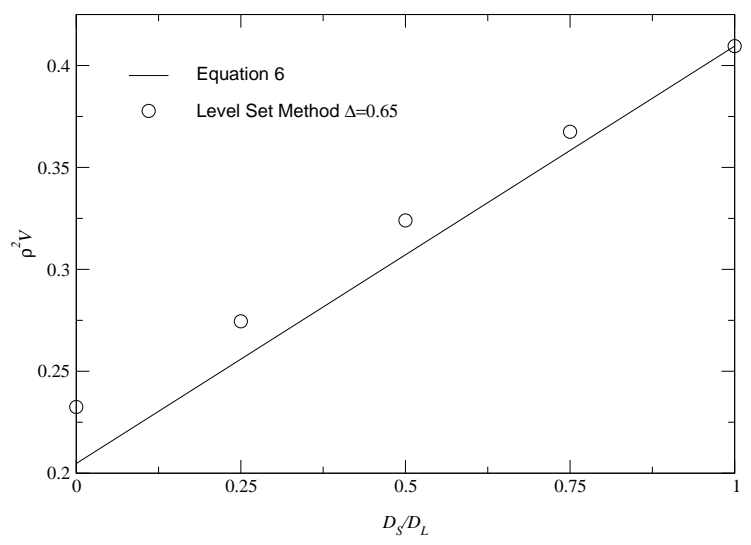

FIG. 3. $\rho^{2} V$ for different values of $D_{S} / D_{L}$. The circles are data from level set simulations at $\Delta=0.65$. The solid line is the theoretical prediction of Barbieri and Langer fitted to the data point at $D_{S} / D_{L}=1$.

In conclusion, the level set method should be considered as a viable alternative to the use of phase-field models. We have used a level set algorithm that can produce accurate calculations of dendritic growth which can be compared favorably with solvability theory as well as time-dependent phase-field model simulations. The level set method can also handle discontinuous material properties easily, which is currently very difficult with the phase-field approach. However, we should note that our implementation is not at all efficient. The practical application of this method to more realistic systems will require some sort of adaptive technique. In the future, we would like to use more computationally efficient implementations of this algorithm and apply these methods to problems in directional solidification, where the ability to simulate unequal diffusivities is of great interest.

We thank Susan Chen and Stanley Osher for useful discussions, Nikolas Provatas for helpful remarks and for providing the adaptive phase-field code used in this work, and Wouter-Jan Rappel for providing the solvability code used to test our simulations. This work has been supported by the NASA Microgravity Research Program, under Grant NAG8-1249. We also acknowledge the support of the National Center for Supercomputing Applications (NCSA) for the use of its computer resources.

[1] R. Almgren, J. Comp. Phys. 106, 337 (1993).

[2] A. R. Roosen and J. E. Taylor, J. Comp. Phys. 114, 113 (1994).

[3] T. Ihle and H. Müller-Krumbhaar, Phys. Rev. E 49, 2972 (1994).

[4] D. Juric and G. Tryggvason, J. Comp. Phys. 123, 127 (1996).

[5] M. E. Glicksman, Materials Science and Engineering 65, 45 (1984).

[6] J. S. Langer, in Chance and Matter, Les Houches Session XLVI, edited by J. V. J. Souletie and R. Stora (North Holland, Amsterdam, 1987), p. 629.

[7] D. A. Kessler, J. Koplik, and H. Levine, Adv. Phys. 37, 255 (1988).

[8] J. B. Collins and H. Levine, Phys. Rev. B 31, 6119 (1985).

[9] G. Caginalp, Arch. Rat. Mech. Anal. 92, 205 (1986).

[10] R. Kobayashi, Physica D 63, 410 (1993).

[11] J. A. Warren and W. J. Boettinger, Acta Metall. Mater. A 43, 689 (1995).

[12] J. S. Langer, Directions in Condensed Matter (World Scientific, Singapore, 1986), p. 164.

[13] G. Caginalp and X. Chen, in On The Evolution Of Phase Boundaries, edited by E. Gurtin and G. McFadden (Springer-Verlag, New York, 1992), Vol. 1, p. 1.

[14] A. Karma and W.-J. Rappel, Phys. Rev. E 53, 3017 (1995); 57, 4323 (1998).

[15] R. Almgren, (unpublished).

[16] S. Osher and J. A. Sethian, J. Comp. Phys. 79, 12 (1988).

[17] B. Merriman, J. K. Bence, and S. J. Osher, J. Comp. Phys. 112, 334 (1994).

[18] M. Sussman, P. Smereka, and S. Osher, J. Comp. Phys. 114, 146 (1994).

[19] T. Y. Hou, Z. Li, S. Osher, and H. Zhao, J. Comp. Phys. 134, 236 (1997).

[20] J. A. Sethian and J. Strain, J. Comp. Phys. 98, 231 (1992).

[21] S. Chen, B. Merriman, S. Osher, and P. Smereka, J. Comp. Phys. 135, 8 (1997).

[22] A. Barbieri and J. S. Langer, Phys. Rev. A 39, 5314 (1989).

[23] D. Peng, B. Merriman, S. Osher, H. Zhao, and M. Kang, UCLA CAM Report 98-25 (1998).

[24] G.-S. Jiang and D. Peng, UCLA CAM Report 97-29 (1997).

[25] N. Provatas, N. Goldenfeld, and J. Dantzig, Phys. Rev. Lett. 80, 3308 (1998); J. Comp. Phys. 148, 265 (1999)

[26] Y.-T. Kim, N. Provatas, N. Goldenfeld, and J. Dantzig, Phys. Rev. E 59, R2546 (1999). 